\title{
Tolerance Estimation of Human Postural Stability against Train Drafts
}

\author{
Hiroharu ENDOH \\ Koji OMINO, Ph.D. \\ Researcher, \\ Laboratory Head, \\ Hiroaki SHIROTO \\ Senior Researcher, \\ Ergonomics Laboratory, Human Science Division
}

\author{
Mitsugu SAWA, Ph.D. \\ Senior Researcher, \\ Ergonomics Laboratory, \\ Human Science Division
}

\author{
Katsuji TANEMOTO \\ Senior Researcher, \\ Aerodynamics Laboratory, \\ Environmental Engineering Division
}

\author{
Yasushi TAKEI \\ Laboratory Head, \\ Architecture Laboratory, Structure Technology Division
}

\begin{abstract}
A wind tunnel experiment was conducted in order to study the effects of train-generated drafts on human postural stability. In the experiment, 29 people were exposed to a transient wind force similar to a draft from a passing train, and the results suggested that both wind speed and wind duration affect postural stability. In this paper, we estimated the tolerance of postural stability against transient wind based on a statistical model and a physical model, and discussed the validity of these models.
\end{abstract}

Keywords: train draft, wind tunnel experiment, posture, wind speed, logistic regression model, rigid model

\section{Introduction}

In recent years, train speeds have been increased for earlier arrival times as part of efforts to improve service to travelers. Meanwhile, the speed of train drafts (the winds generated when trains pass by station platforms) increases in proportion to the train speed. JR companies have therefore deployed adequate safety measures such as the installation of platform doors and aerodynamic optimization of train nose configurations. Since train speeds are expected to increase further in the future, it is essential to carry out basic research on the effects of train wind on passengers waiting on station platforms.

In the past, many field investigations have been conducted, and the results have suggested that train drafts exhibit characteristic waveforms such as the triangular waves observed when the train nose passes [1]. However, there have been no studies on the effects of such wind on human beings. Although Penwarden proposed a table describing the effects of natural wind on human beings based on daily experience and observation [2], the table did not describe the effects of wind waveforms. We therefore conducted a wind tunnel experiment to investigate the effects of winds with triangular waveforms on human beings. This paper outlines the experiment, and the results are used to analyze the effects of wind peak and wind duration on human beings (with a focus on postural stability) by employing a statistical model and a physical model.

\section{Wind tunnel experiments}

\subsection{Experimental method}

We conducted experiments in the low-noise wind tunnel of the Railway Technical Research Institute. An outline of the experimental method is given below.

Experimental apparatus: The louver shown in Fig. 1 was arranged in the wind tunnel in order to enable subjects to experience a triangular wind. The louver is designed to withstand wind speeds of $30 \mathrm{~m} / \mathrm{s}$, and has twenty blades that can be opened and closed together by rotating a handle manually. The louver opens fully when the blades rotate by 45 degrees, and closes completely when they rotate by 90 degrees. A triangular wind blowing toward the subjects was generated by rotating the handle while a steady wind was blowing in the tunnel.

Wind speed condition: The peak of the triangular wind to which the subjects were exposed increased in proportion to the speed of the steady wind blowing in the tunnel. The speed of the steady wind was increased in six increments from $5 \mathrm{~m} / \mathrm{s}$ to $30 \mathrm{~m} / \mathrm{s}$ at $5 \mathrm{~m} / \mathrm{s}$ intervals. For each wind speed level, the duration of the triangular wind was set at three levels $(0.5 \mathrm{~s}, 1.0 \mathrm{~s}$ and $2.0 \mathrm{~s})$ by controlling the opening and shutting time of the louver blade. The test order of the wind duration level was randomized and was not revealed to the subjects. However, if a subject could not hold his/her posture in all three duration conditions at one wind speed level, the next level was not implemented.

Posture: Subjects stood naturally: They widened their legs to shoulder width and looked straight ahead. When passengers are exposed to train drafts from behind, there is a danger of them being drawn down onto the rail track. 


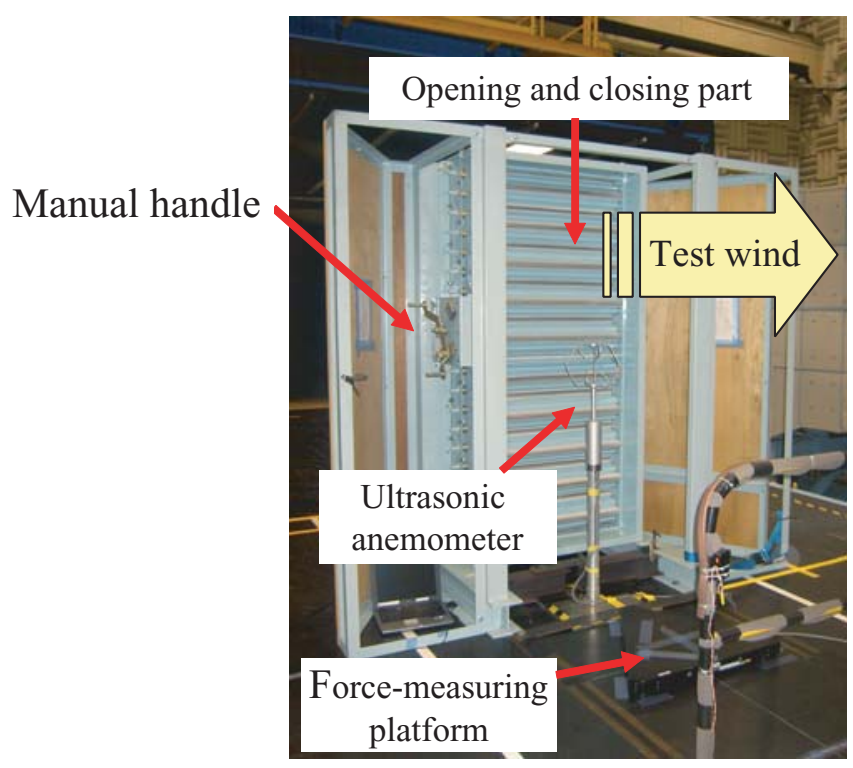

Fig. 1 Wind conversion louver (steady wind into transient wind)

In addition, people generally exhibit lower stability to forces in the front-rear direction than those in the lateral direction. To consider on the safe side, the subjects were exposed to wind from behind in the experiments.

Subjects: A total of 29 healthy adults (13 males and 16 females) participated in the experiments. Their ages ranged from 18 to 64 (mean: 38.0, SD: 13.0); their height from $1.51 \mathrm{~m}$ to $1.81 \mathrm{~m}$ (mean: $1.64 \mathrm{SD}$ : 0.09); their weight from $42.6 \mathrm{~kg}$ to $98.2 \mathrm{~kg}$ (mean: 60.3, SD: 11.6); and their shoe size from $0.225 \mathrm{~m}$ to $0.280 \mathrm{~m}$ (mean: 0.248 , SD: 0.016).

Measurement items: The wind speed was measured using an ultrasonic anemometer (Kaijo, SA200) arranged on the windward side of the subjects. The posture of the subjects exposed to the wind was recorded using a video camera. The floor reaction force was measured using a force-measuring platform (ANIMA, MG100).

\subsection{Results}

\subsubsection{Wind peak and duration}

Since the speed of the steady wind blowing in the wind tunnel fluctuated to some extent, the measured peak values of the triangular wind were different even in the same test wind speed conditions. In addition, the duration of the triangular wind was also different from the condition values because of the manual operation of the louver. The peak value and the duration were read off from each measured wind speed. In this study, to decide the duration of measured wind speed in a regular manner, we identified it as the width of the base of an isosceles triangle with the same peak as the measured wind speed and with an area formed by a wind speed wave whose speed was more than $10 \%$ of the measured peak value. An example of the approximation is shown in Fig. 2, which depicts an approximated isosceles triangle with a wind peak (height) of $19.4 \mathrm{~m} / \mathrm{s}$ and a wind duration (width) of $1.27 \mathrm{~s}$. The ranges of peak and duration obtained in the experiments

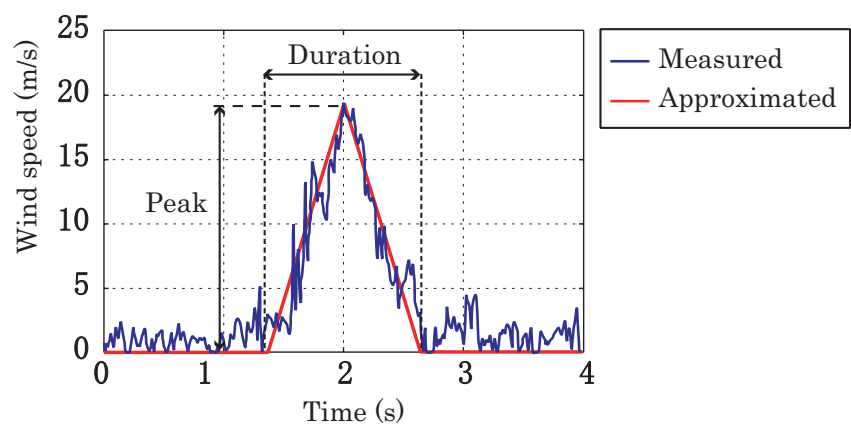

Fig. 2 Measured wind speed

were from $1.37 \mathrm{~m} / \mathrm{s}$ to $38.8 \mathrm{~m} / \mathrm{s}$ and from $0.30 \mathrm{~s}$ to $3.0 \mathrm{~s}$, respectively.

\subsubsection{Results of Posture Observation}

The following three posture types were observed when the subjects experienced the various triangular winds (Fig. 3):

Posture 1: Rotational motion around the ankles with the heels in contact with the floor

Posture 2: Heel-off motion

Posture 3: Heel-off and stepping motion

The difference between Posture 1 and Posture 2 is

Example of Posture 1 (peak $4.5 \mathrm{~m} / \mathrm{s}$, duration $1.6 \mathrm{~s}$ )

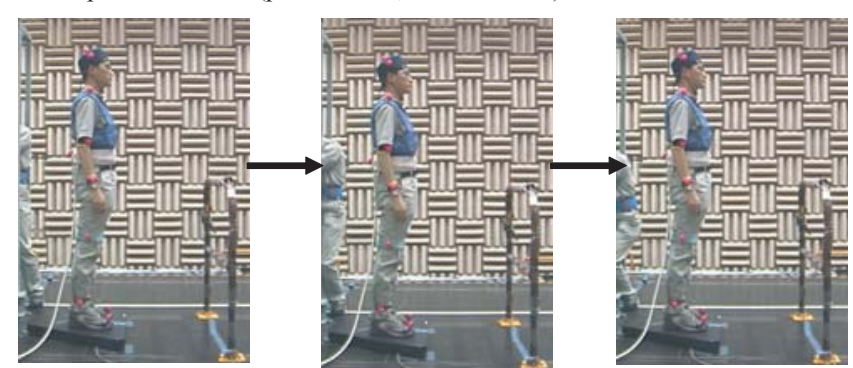

Example of Posture 2 (peak $20.8 \mathrm{~m} / \mathrm{s}$, duration $2.0 \mathrm{~s}$ )

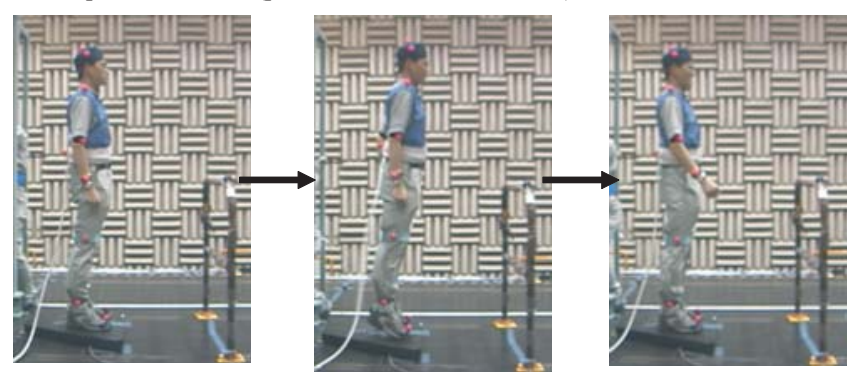

Example of Posture 3 (peak $31.5 \mathrm{~m} / \mathrm{s}$, duration $1.9 \mathrm{~s}$ )

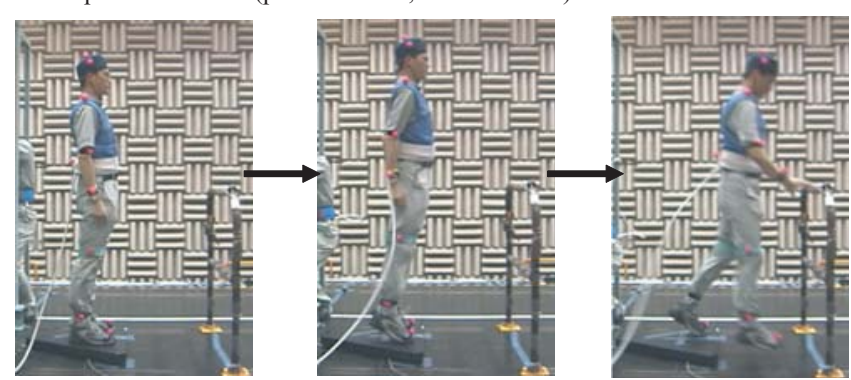

Fig. 3 The three posture types during wind exposure 


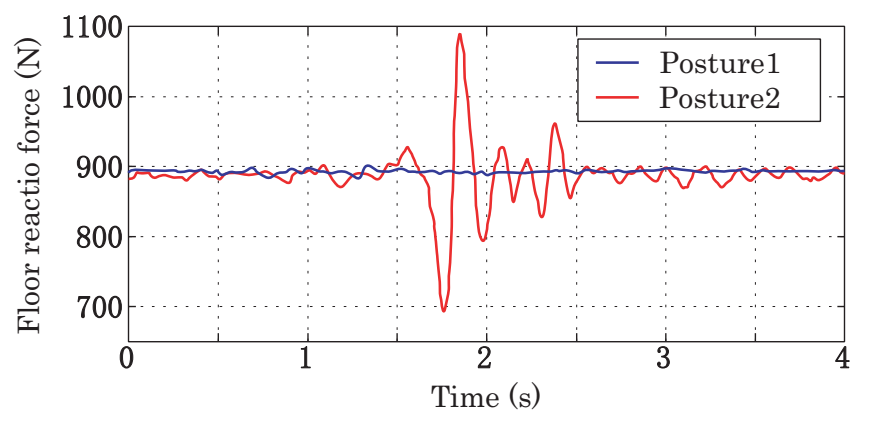

Fig. 4 Examples of floor reaction force waveform for Posture 1 and Posture 2

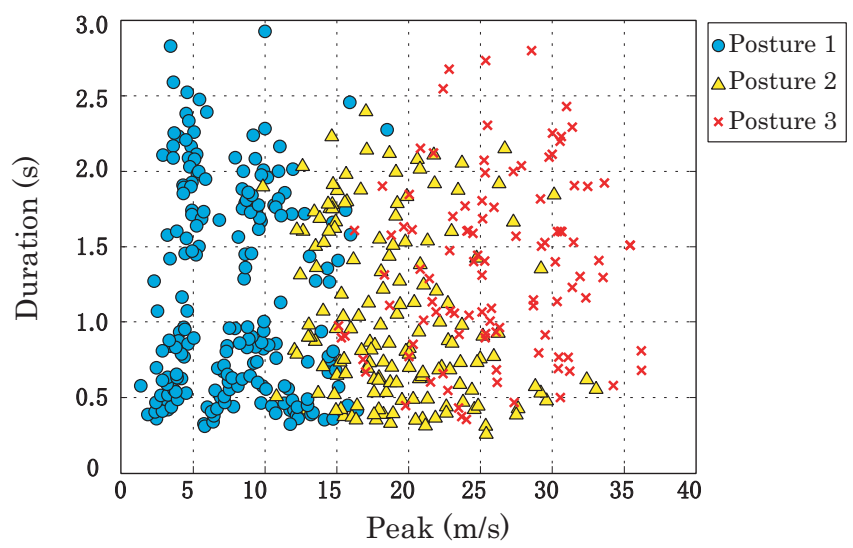

Fig. 5 Experimental results for posture type

whether the heels are off the floor. However, it was difficult to distinguish these two posture types by video observation. Accordingly, we utilized the measured floor reaction force, and an example of the measured data is shown in Fig. 4. In the case of Posture 2, when the heels lift from the floor and join it again, the floor is subject to a relatively large impact. We identified the posture type as Posture 2 if the variation in the vertical component of the floor reaction force was more than $10 \%$ of the total weight of the subject on the force-measuring platform. Figure 5 shows the results of the posture types depending on the measured peaks (horizontal axis) and durations (vertical axis) of the triangular wind in the experiments. The marker type in Fig. 5 corresponds to the observed posture type for each test. As the wind speed increases, the posture type changes in the order of Posture 1, Posture 2, Posture 3. The boundary between Posture 1 and Posture 2 is comparatively clear and is not influenced by the wind duration. In contrast, the boundary between Posture 2 and Posture 3 is unclear and is influenced by the wind duration. As the duration is shorter, more Posture 2 types are observed in the higher wind speed region.

\section{Estimation of posture type using a logistic regression model}

The occurrence probability of Posture 1, Posture 2 and Posture 3 when a subject is exposed to a triangular wind with a wind peak of $U_{p}(\mathrm{~m} / \mathrm{s})$ and a wind duration of $T_{d}(\mathrm{~s})$ can be expressed by a logistic regression model as fol- lows:

$$
\begin{aligned}
& P_{1}=\frac{e^{a_{1} U_{p}+b_{1} T_{d}+c_{1}}}{1+e^{a_{1} U_{p}+b_{1} T_{d}+c_{1}}+e^{a_{2} U_{p}+b_{2} T_{d}+c_{2}}} \\
& P_{2}=\frac{e^{a_{2} U_{p}+b_{2} T_{d}+c_{2}}}{1+e^{a_{1} U_{p}+b_{1} T_{d}+c_{1}}+e^{a_{2} U_{p}+b_{2} T_{d}+c_{2}}} \\
& P_{3}=\frac{1}{1+e^{a_{1} U_{p}+b_{1} T_{d}+c_{1}}+e^{a_{2} U_{p}+b_{2} T_{d}+c_{2}}}
\end{aligned}
$$

where $P_{1}, P_{2}$ and $P_{3}$ are the occurrence probabilities of Posture 1, Posture 2 and Posture 3 respectively, and $a_{1}$, $b_{1}, c_{1}, a_{2}, b_{2}$ and $c_{2}$ are model parameters. Using the maximum likelihood method, we estimated $a_{1}, b_{1}, c_{1}, a_{2}, b_{2}$ and $c_{2}$ at $-1.05,-1.36,18.7,-0.27,-1.01$ and 7.6 respectively. Figure 6 shows the estimated $P_{1}, P_{2}, P_{3}$ values against wind speed in the cases of a wind duration of $0.5 \mathrm{~s}$ and $2.0 \mathrm{~s}$. As the wind speed increases from $10 \mathrm{~m} / \mathrm{s}$ to $15 \mathrm{~m} / \mathrm{s}$, the posture type rapidly changes from Posture 1 to Posture 2 , and in this wind speed region, $P_{1}$ and $P_{2}$ do not depend on the wind duration. In contrast, in the wind speed region where the posture type changes from Posture 2 to Posture $3, P_{2}$ and $P_{3}$ both depend on the wind duration. For example, when the wind duration is $0.5 \mathrm{~s}$, the wind speed at which $P_{3}$ becomes 0.5 is $26.4 \mathrm{~m} / \mathrm{s}$; on the other hand, when the wind duration is $2.0 \mathrm{~s}$, the corresponding wind speed is $20.8 \mathrm{~m} / \mathrm{s}$.

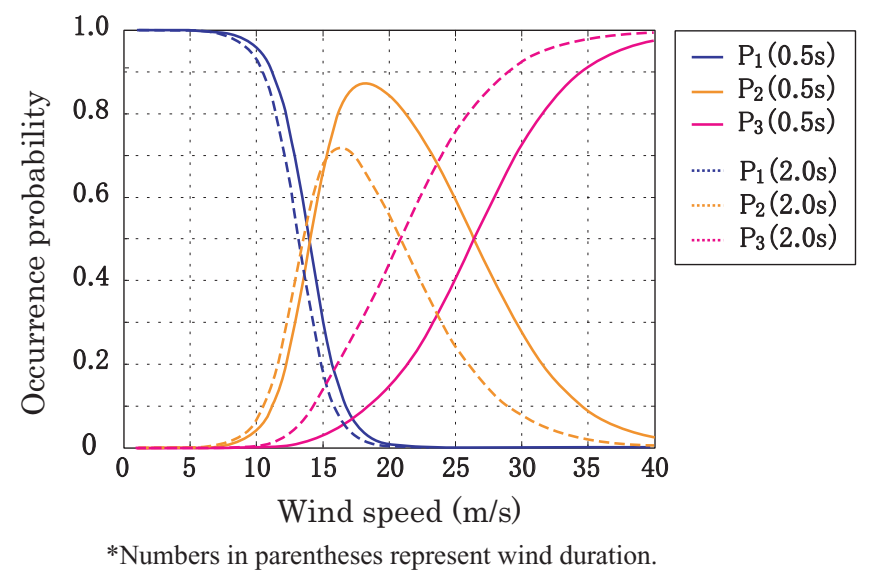

Fig. 6 Occurrence probability of posture type estimated using the logistic regression model

\section{Estimation of posture type using a rigid model}

In the preceding chapter, the occurrence probability of each posture type was estimated using a statistical model. Since the model parameters were estimated based on experimental results reflecting the characteristics of the subjects, high-accuracy estimation of the general characteristics of postural stability can be achieved by conducting more experiments and acquiring more data. On the other hand, since it is considered that individual attributes such as height and weight influence postural stability, high-accuracy estimation of the individual characteristics of postural stability is also important when safety assessment of train drafts is conducted. In addition, in order to interpret the experimental results properly, it is necessary to analyze them from a physical point of view as well as a statistical 
point of view. Accordingly, we examined the influence of triangular winds on human postural stability from a physical point of view and inspected the feasibility of estimating the posture type during wind exposure on an individual level.

\subsection{Rigid model}

From the observation results outlined in Chapter 2, it was confirmed that as the wind speed increased, the posture type changed in the order of Posture 1, Posture 2, Posture 3. In this study, the wind speed corresponding to the border between Posture 1 and Posture 2 is referred to as the maximum standstill wind speed, and that corresponding to the border between Posture 2 and Posture 3 is the tolerance limit wind speed. To estimate these two wind speeds, we modeled a person as a rigid body with one rotational degree of freedom around the tips of the toes based on the observation results outlined in Chapter 2.

The proposed model is shown in Fig. 7, and consists of two boards: one with a uniform thickness (corresponding to human limbs and a human trunk), and the other with no thickness (corresponding to human feet). The height of the model's center of gravity is $55 \%$ of its height, and the horizontal position of its center of gravity is at a position $60 \%$ of the shoe size from the tips of the toes. The motion equation of this model is as follows:

$$
J \ddot{\theta}+M g L_{0} \cos \left(\theta+\theta_{0}\right)=F_{u} L_{0} \sin \left(\theta+\theta_{0}\right)
$$

where $\theta$ is the angle between the floor and the bottom of the foot; $J$ is the moment of inertia around the tips of the toes; $M$ is the model mass; $L_{0}$ is the distance of point $\mathrm{P}$ and the model's center of gravity $C_{g}$; and $\theta_{0}$ is the angle between line $\mathrm{PCg}$ and the bottom of the foot. $F_{u}$ is the wind force to which the model is exposed, and is assumed to be described as follows:

$$
F_{u}(t)=\frac{1}{2} \rho C_{d} S_{u} U^{2}(t)
$$

where $S_{u}$ is the projected area of the model's frontal plane; $\rho$ is the air density; and $C_{d}$ is the drag coefficient. In this study, $\rho$ and $C_{d}$ were set at $1.23 \mathrm{~kg} / \mathrm{m}^{3}$ and 1.0 respectively with reference to the preceding study [3]. The maximum standstill wind speed of the model is provided in (4) by assuming $\theta=0$ and $\ddot{\theta}=0$, and is described as follows:

$$
U_{b}=\sqrt{\frac{2 M g}{\rho C_{d} S_{u} \tan \theta_{0}}}
$$

If the peak of the wind speed is greater than $U_{b}$, rotary motion occurs in the model regardless of the waveform of
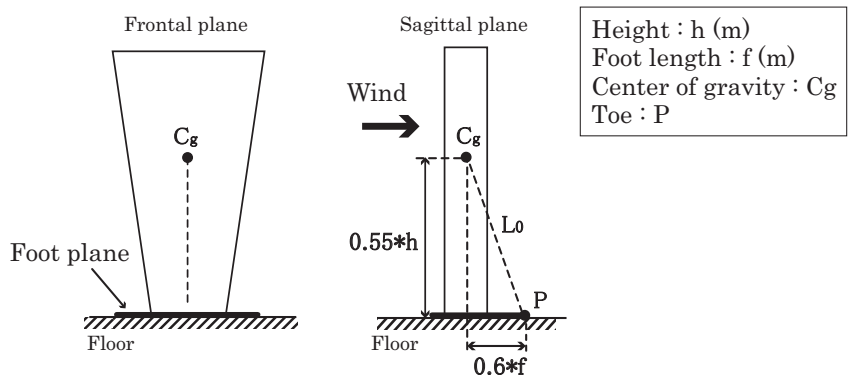

Fig. 7 Rigid model the wind speed. On the other hand, in order to estimate whether the model falls, numerical calculation of (4) is necessary because it depends not only on the peak but also on the waveform of the wind speed.

\subsection{Comparison between the experimental results and the rigid model results}

We estimated the posture type of subjects in each test using (4) to (6), in which the values of measured wind speed and measured dimensions (weight, height, shoe size and projected area of subjects) were used. The model results can be related to the posture types described in Chapter 2 as follows:

Posture 1: Model does not rotate.

Posture 2: Model rotates but does not fall to the floor.

Posture 3: Model falls to the floor.

Numerical calculation of (4) was conducted using the Runge-Kutta method of order 4.

Tables 1 to 3 show the relationship between the experimental results and the rigid model results. The model results corresponded to the experimental results at a ratio of $78.5 \%$ ( $81.0 \%$ for males, $76.4 \%$ for females). In this study, the ratio of the correspondence between the experimental results and the model results was defined as the estimation accuracy. Figure 8 shows the relationship between the estimation accuracy and the age of the subjects, and indicates that the estimation accuracy of the model is relatively high regardless of age. It is therefore suggested that the proposed model can estimate the posture type accurately on an individual level when subjects are exposed to triangular winds.

As shown in Tables 1 to 3, estimation errors were seen mainly in two cases: one involved experimental results suggesting Posture 2, when in fact the result was Posture 1, and the other involved results suggesting Posture 3 when in fact it was Posture 2. The former case was seen comparably in males and females. The cause of the error is considered to be the assumption of the model's rotation axis, where the tips of the toes represent the only rotational degree of freedom in the model. In order to increase the estimation accuracy, other degrees of freedom such as the metatarsal joint should be introduced into the model. The latter case was seen especially in females. In the case of females under psychological influence, it is very likely that they were unable to maintain posture even at wind speeds lower than the tolerance limit estimated by the model. Thus, it is considered that this error is due

Table 1 Cross table showing experimental results and model results (male and female)

\begin{tabular}{|c|c|c|c|}
\hline Male \& female & \multicolumn{3}{|c|}{ Model results } \\
\hline $\begin{array}{c}\text { Experimental } \\
\text { results }\end{array}$ & Posture 1 & Posture 2 & Posture 3 \\
\hline Posture 1 & $212(44.2 \%)$ & $2(0.4 \%)$ & $0(0 \%)$ \\
\hline Posture 2 & $58(12.1 \%)$ & $95(19.8 \%)$ & $9(1.9 \%)$ \\
\hline Posture 3 & $4(0.8 \%)$ & $30(6.3 \%)$ & $70(14.6 \%)$ \\
\hline \\
*Numerical values in the table represent the number of trials. \\
*Numbers in parentheses represent the figure as a percentage of all trials (480).
\end{tabular}

QR of RTRI, Vol. 50, No. 1, Feb. 2009 
Table 2 Cross table showing experimental results and model results (males)

\begin{tabular}{|c|c|c|c|}
\hline Male & \multicolumn{3}{|c|}{ Model results } \\
\hline $\begin{array}{c}\text { Experimental } \\
\text { results }\end{array}$ & Posture 1 & Posture 2 & Posture 3 \\
\hline Posture 1 & $99(43.8 \%)$ & $1(0.4 \%)$ & $0(0 \%)$ \\
\hline Posture 2 & $31(13.7 \%)$ & $47(20.8 \%)$ & $5(2.2 \%)$ \\
\hline Posture 3 & $1(0.4 \%)$ & $5(2.2 \%)$ & $37(16.4 \%)$ \\
\hline
\end{tabular}

*Numerical values in the table represent the number of trials.

*Numbers in parentheses represent the figure as a percentage of all trials involving male (226)

Table 3 Cross table showing experimental results and model results (females)

\begin{tabular}{|c|c|c|c|}
\hline Female & \multicolumn{3}{|c|}{ Model results } \\
\hline $\begin{array}{c}\text { Experimental } \\
\text { results }\end{array}$ & Posture 1 & Posture 2 & Posture 3 \\
\hline Posture 1 & $113(44.5 \%)$ & $1(0.4 \%)$ & $0(0 \%)$ \\
\hline Posture 2 & $27(10.6 \%)$ & $48(18.9 \%)$ & $4(1.6 \%)$ \\
\hline Posture 3 & $3(1.2 \%)$ & $25(9.8 \%)$ & $33(13.0 \%)$ \\
\hline
\end{tabular}

**Numerical values in the table represent the number of trials.

*Numbers in parentheses represent the figure as a percentage of all trials involving females (254 trials).

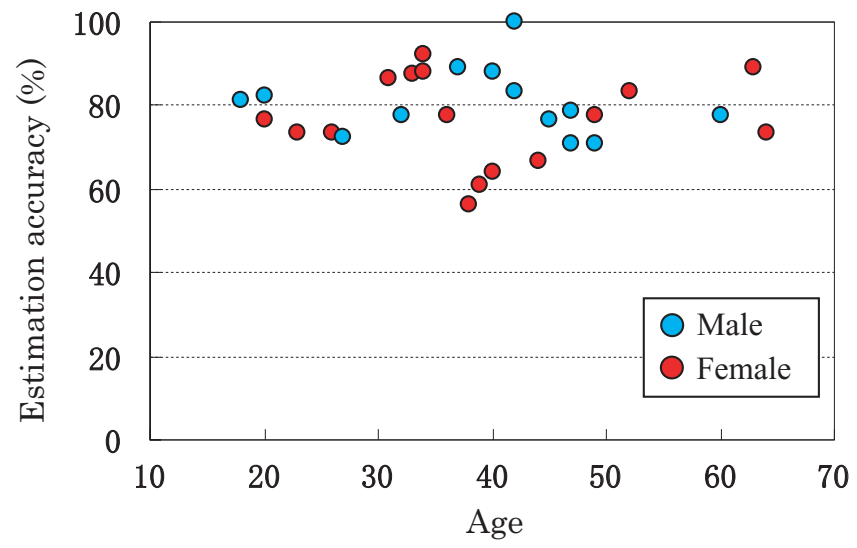

Fig. 8 Relationship between age and estimation accuracy

to the assumption that the wind speed at which the model falls to the floor corresponds to the wind speed at which the subjects cannot maintain posture. Improving those two estimation errors is a task for the future.

\section{Analytical solution of tolerance limit wind speed}

In the previous chapter, it was confirmed that the proposed model expressed by (4) and (5) was effective in the estimation of posture type when a person was exposed to triangular winds. In order to clarify the relationship between the model parameters and the tolerance limit wind speed (corresponding to the wind speed at which the model falls to the floor), this chapter derives an analytical solution for (4) by assuming the waveform of the wind speed to be an isosceles triangle.

By applying the first-order Taylor polynomial approxi- mation to $\cos \left(\theta+\theta_{0}\right)$ and $\sin \left(\theta+\theta_{0}\right)$, and by considering that $\left(\cos \theta_{0}\right) \cdot \theta<<\sin \theta_{0}$ around $\theta=0$, (4) can be approximated as follows:

$$
J \ddot{\theta}+M g L_{0}\left(\cos \theta_{0}-\sin \theta_{0} \cdot \theta\right)=F_{u} L_{0} \sin \theta_{0}
$$

The waveform of the wind speed is assumed to be an isosceles triangle as shown in Fig. 9. Since (7) does not consider the force between the model and the floor, it is assumed that the wind speed starts from the maximum standstill wind speed at which the model begins to move. The isosceles triangle $U(t)$ shown in Fig. 9 is described as follows:

$$
\begin{aligned}
U(t) & =\left[U_{p}+2 \frac{U_{p}}{T_{d}}\left(t-T_{1}\right)\right] \cdot\left[H(t)-H\left(t-T_{1}\right)\right] \\
& +\left[U_{p}-2 \frac{U_{p}}{T_{d}}\left(t-T_{1}\right)\right] \cdot\left[H\left(t-T_{1}\right)-H\left(t-T_{2}\right)\right] \\
T_{1}=\frac{T_{d}}{2}\left(1-\frac{U_{b}}{U_{p}}\right), \quad T_{2}=T_{d}\left(1-\frac{U_{b}}{2 U_{p}}\right) & \\
H(t) & = \begin{cases}1 & (t \geq 0) \\
0 & (t<0)\end{cases}
\end{aligned}
$$

where $U_{p}$ is the wind speed peak and $T_{d}$ is the wind duration. By solving the differential equation through the substitution of (5) and (8) into (7), $\theta(t)$ after wind exposure $\left(t>T_{2}\right)$ is described as follows:

$$
\begin{array}{r}
\theta(t)=\frac{1}{\tan \theta_{0}}\left[1+\frac{\alpha}{\beta}\left(1+\frac{\alpha}{\beta}-2 \alpha e^{-(\alpha-1) \frac{\beta}{\alpha}}-\frac{\alpha}{\beta} e^{-(2 \alpha-1) \frac{\beta}{\alpha}}\right) e^{L_{s} t}\right. \\
\left.+\frac{\alpha}{\beta}\left(-1+\frac{\alpha}{\beta}+2 \alpha e^{(\alpha-1) \frac{\beta}{\alpha}}-\frac{\alpha}{\beta} e^{(2 \alpha-1) \frac{\beta}{\alpha}}\right) e^{-L_{s} t}\right] \\
\alpha=\frac{U_{p}}{U_{b}}, \quad \beta=\frac{T_{d} L_{s}}{2}, \quad L_{s}=\sqrt{\frac{M g L_{0} \sin \theta_{0}}{J}}
\end{array}
$$

Since (7) does not consider the force between the floor and the model, $\theta \rightarrow \infty$ when $t \rightarrow \infty$ if the model falls to the floor, and $\theta \rightarrow-\infty$ when $t \rightarrow \infty$ if the model returns to its initial position rather than falling. The sign of $\theta$ depends on the coefficients of $e^{L_{s} t}$ because $L_{s}>0$. Then, the tolerance limit wind speed of the model can be ex-

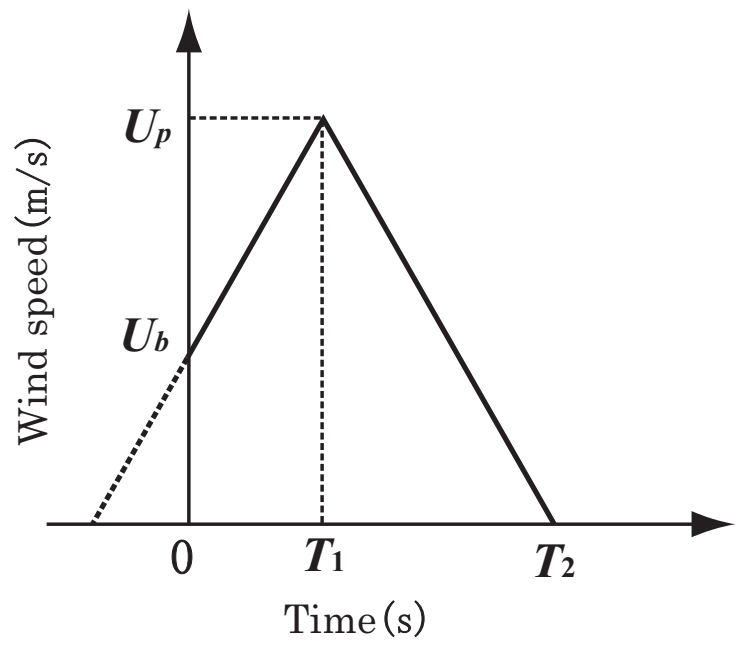

Fig. 9 Isosceles triangular wind speed 
pressed as a curve that satisfies the following equation:

$$
F(\alpha, \beta)=1+\frac{\alpha}{\beta}-2 \alpha e^{-(\alpha-1) \frac{\beta}{\alpha}}-\frac{\alpha}{\beta} e^{-(2 \alpha-1) \frac{\beta}{\alpha}}=0
$$

where the model does not fall to the floor when $F<0$, and does when $F>0$. Examples of the maximum standstill wind speed and tolerance limit wind speed for isosceles triangle wind are shown in Fig. 10 (a). For comparison, numerical calculation results of the tolerance limit wind speed are also shown. In the numerical calculation, the peak of wind with a fixed duration was increased at 0.01 $\mathrm{m} / \mathrm{s}$ intervals until the model fell to the floor, and the wind speed peak immediately before falling was defined as the tolerance limit wind speed. As shown in Fig. 10 (a), the analytical solution represented by (13) and the numerical calculation results of (7) were almost the same; this indicates that few errors of Tailor approximation are applied to (7). In addition, as to the posture type estimated using (7), 97\% of the approximated wind speeds by isosceles triangle brought the same results as the measured wind speed.

The maximum standstill wind speeds for the subjects participating in the experiment were estimated as ranging from $15.3 \mathrm{~m} / \mathrm{s}$ to $19.5 \mathrm{~m} / \mathrm{s}$. Higher tolerance limit wind speeds were estimated as the wind duration became shorter. For example, wind speeds were estimated from $18.9 \mathrm{~m} / \mathrm{s}$ to $24.5 \mathrm{~m} / \mathrm{s}$ when the wind duration was $2.0 \mathrm{~s}$, and from $27.2 \mathrm{~m} / \mathrm{s}$ to $35.7 \mathrm{~m} / \mathrm{s}$ when the wind duration was $0.5 \mathrm{~s}$.

$\alpha$ and $\beta$ in (13) are the values given by standardizing the wind speed peak and wind duration by the dimensions of the model. Although in the $U_{p}-T_{d}$ space the maximum standstill wind speed line and the tolerance limit wind speed line depend on the dimensions of the model, in the $\alpha-\beta$ space both these wind speed lines are determined regardless of the dimensions of the model. The maximum standstill wind speed line, the tolerance limit wind speed line and the experimental results of posture type are shown in the $\alpha-\beta$ space in Fig. 10 (b). The figure indicates that the overall trend of posture type changes can be estimated accurately using the maximum standstill wind speed line $(\alpha=1)$ and the tolerance limit wind speed line.
This chapter therefore confirms that the posture type of a person during triangular wind exposure can be estimated accurately by approximating the measured triangular wind speed using the isosceles triangular wind speed, and by inputting the wind peak, the wind duration and the dimensional data of the person into (12) and (13).

\section{Comparison between the results of the logistic regression model and the rigid model}

In this chapter, we compare the results of the occurrence probability estimated by the logistic regression model to the results of the maximum standstill wind speed and the tolerance limit wind speed estimated by the rigid model.

The maximum standstill wind speed is the speed at which the heels begin to lift from the floor, and corresponds to the probability of becoming Posture 2 or Posture $3\left(P_{2}+P_{3}\right)$ in the logistic regression model. The tolerance limit wind speed corresponds to the probability of becoming Posture $3\left(P_{3}\right)$. For comparison, contour lines showing a probability of 0.5 for $P_{2}+P_{3}$ and $P_{3}$ are shown in Fig. 10 (a).

A probability of 0.5 for $P_{2}+P_{3}$ corresponds to a wind peak of $14.0 \mathrm{~m} / \mathrm{s}$ when the wind duration is $0.5 \mathrm{~s}$ and to a wind peak of $13.2 \mathrm{~m} / \mathrm{s}$ when the wind duration is $2.0 \mathrm{~s}$; the difference in the wind peak due to the wind duration is small. This result agrees with that of the rigid model, in which the maximum standstill wind speed is constant regardless of the wind duration when the dimensional data of the rigid model is fixed. The contour line of a probability of 0.5 for $P_{2}+P_{3}$ seems different from the maximum standstill wind speed line. As mentioned in Section 4.2 , this difference is due to the location of the rotational axis of the rigid model, and therefore does not contradict the mechanical interpretation.

The contour line of a probability of 0.5 for $P_{3}$ depends heavily on the wind duration. This result agrees with that of the rigid model, in which the tolerance limit speed depends heavily on the wind duration even when the dimensional data of the rigid model is fixed. The contour

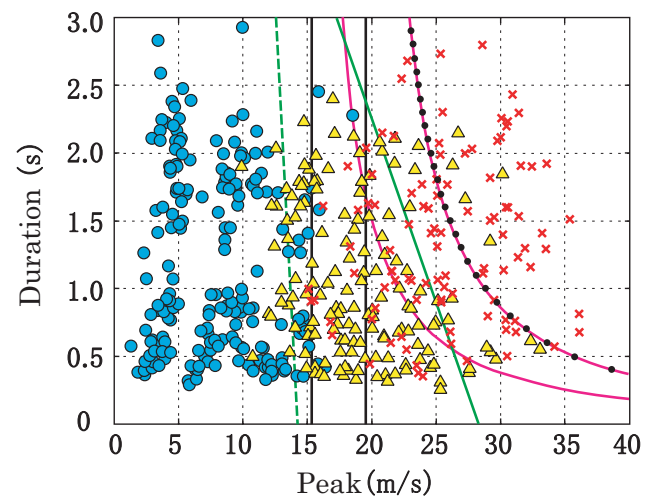

(a) Peak-duration space

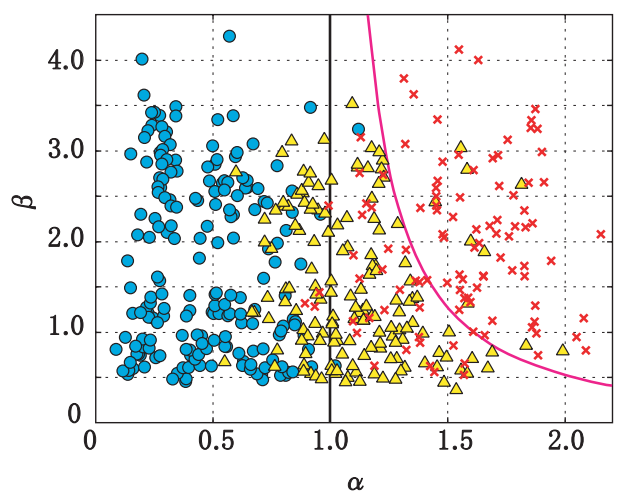

(b) Nomalized space by model dimension

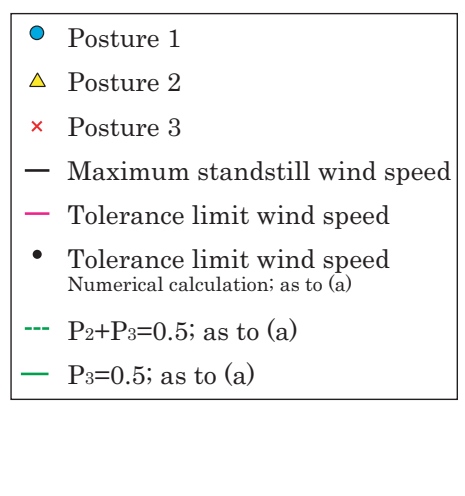

um line and the minimum line among the subjects in (a).

Fig. 10 Results of posture experiments (logistic regression model and rigid model) 
line of a probability of 0.5 for $P_{3}$ is located between the maximum standstill wind speed line and the tolerance limit wind speed line in the wind duration region over $0.5 \mathrm{~s}$. On the other hand, in the wind duration region below $0.5 \mathrm{~s}$, a large difference is seen between the results of the logistic regression model and those of the rigid model. It is necessary to inspect the validity of both models by acquiring more experimental data in the future.

\section{Conclusion}

In order to investigate the effects of triangular winds on human postural stability, we conducted wind tunnel experiments in which subjects were exposed to triangular winds with peaks ranging from $1.37 \mathrm{~m} / \mathrm{s}$ to $38.8 \mathrm{~m} / \mathrm{s}$ and with durations ranging from $0.30 \mathrm{~s}$ to $3.0 \mathrm{~s}$. We then analyzed the experimental results based on a statistical model and a physical model. The findings obtained in the study are described below:

(1) In the wind tunnel experiments, motion in which subjects' heels began to lift from the floor and motion in which they took a step (i.e., could not maintain posture) were observed as wind speeds increased.

(2) As a result of logistic regression analysis, the triangular wind speed at which a subject's heels lifted from the floor with a probability of 0.5 was estimated at around $13 \mathrm{~m} / \mathrm{s}$ to $14 \mathrm{~m} / \mathrm{s}$, and more little relation to the wind duration. In addition, the triangular wind speed at which a subject could not maintain posture with a probability of 0.5 was estimated as $26.4 \mathrm{~m} / \mathrm{s}$ when the wind duration was $0.5 \mathrm{~s}$, and as $20.8 \mathrm{~m} / \mathrm{s}$ when the wind duration was $2.0 \mathrm{~s}$. It was therefore confirmed that it became easier for subjects to maintain posture as the wind duration became shorter.

(3) By approximating a person using a simple rigid model, we proposed a method that predicts the posture type of a person experiencing a triangular wind. The rigid model results corresponded to the experimental results with a ratio of $78.5 \%$. In addition, in terms of the age range of the subjects (from 18 to 64 ), almost no influence was found on the estimation accuracy of the rigid model. As to sex, the estimation accuracy for females was slightly lower than that for males.

(4) We calculated an analytical solution for the tolerance limit wind speed of the proposed rigid model when it was exposed to isosceles triangular wind. Under this analytical solution, once the wind peak, wind duration and dimensional information of the model are decided, it is possible to accurately estimate the tolerance limit wind speed on an individual level.

(5) Through comparison between the results of the logistic regression model and those of the rigid model, it was confirmed that the findings outlined in (2) could be compatibly interpreted from a mechanical point of view. However, for triangular wind with durations of less than $0.5 \mathrm{~s}$, the results of the logistic regression model differed largely from those of the rigid model. For the statistical model, we plan to continue gathering data to enable inspection of its validity, and for the rigid model we will try to reduce the estimation error as described in Section 4.2. We also plan to advance an evaluation model that will be developed from both a statistical and a physical point of view, and to utilize it as a means of evaluating the safety of train-generated drafts.

\section{References}

[1] Tanemoto, K., Kajiyama, H., "Train Draft and Pressure Variation on a Platform," RTRI Report, Vol. 17, No. 11, pp. 53-56, 2003 (in Japanese).

[2] Omino, K., Shiroto, H. et al., "Effect of Train Draft on Passengers on Station Platform," RTRI Report, Vol. 20, No. 3, pp. 41-46, 2006 (in Japanese).

[3] Murakami, S., Deguchi, K. et al., "Measurement of Drag Force on People Walking in Wind Tunnel Floor," Proceedings of the 6th National Symposium on Wind Engineering, pp.115-122, 1980 (in Japanese). 